\title{
Argumentation and qualitative probabilistic reasoning using the kappa calculus
}

\author{
Valentina Tamma \& Simon Parsons \\ Department of Computer Science, University of Liverpool \\ Chadwick Building, Liverpool, L69 7ZF, UK \\ \{V.A.M.Tamma, S.D.Parsons\}@csc.liv.ac.uk
}

\begin{abstract}
This paper presents the $Q R K$ system for reasoning under uncertainty, which combines the building of logical arguments for formulae with infinitesimal probabilities of the kind handled by the kappa calculus. Each constituent of an argument has an associated $\kappa$-value which captures belief in that component, and these values are combined when arguments are constructed from the components. The paper is an extension of our previous work on systems of argumentation which reason with qualitative probabilities, providing a finer-grained approach to handling uncertainty.
\end{abstract}

\section{Introduction}

In the last few years there have been a number of attempts to build systems for reasoning under uncertainty that are of a qualitative nature - that is they use qualitative rather than numerical values, dealing with concepts such as increases in belief and the relative magnitude of values. Three main classes of system can be distinguished - systems of abstraction, infinitesimal systems, and systems of argumentation. In systems of abstraction, the focus is mainly on modelling how the probability of hypotheses changes when evidence is obtained. Such systems provide a qualitative abstraction of probabilistic networks, known as qualitative probabilistic networks (QPNs), which is sufficient for planning [14], explanation [3] and prediction [11] tasks. Infinitesimal systems deal with beliefs that are very nearly 1 or 0 , providing formalisms that handle order of magnitude probabilities. Such systems may be used for diagnosis [2] and have been extended with infinitesimal utilities to give complete decision theories $[12,15]$. Systems of argumentation are based on the idea of constructing logical arguments for and against formulae. Such systems have been applied to problems such as diagnosis, protocol management and risk assessment [5], as well as handling inconsistent information [1], and providing a framework for default reasoning $[4,9]$.

In this paper we provide a hybridisation of infinitesimal systems and systems of argumentation, by defining a system of argumentation which uses order of magnitude probabilities, in particular the values manipulated by the kappa calculus. 


\section{Kappa calculus}

The kappa calculus is a formalism that makes it possible to handle order of magnitude probabilities, representing a state of belief by means of a ranking $\kappa$ that maps propositions into class of ordinals. This mapping is such that:

$$
\begin{aligned}
\kappa(\text { true }) & =0 \\
\kappa(\phi \vee \psi) & =\min (\kappa(\phi), \kappa(\psi))
\end{aligned}
$$

According to the kappa calculus, a proposition $\alpha$ is believed to degree $s$, if $\kappa(\neg \alpha)=s$; is disbelieved to degree $s$ if $\kappa(\alpha)=s$; and is uncommitted if $\kappa(\alpha)=$ $\kappa(\neg \alpha)=0$. When accommodating disbelieved evidence, the choice about which beliefs have to be retracted depends on their strength.

The $\kappa$ ranking also has the following properties, analogous to the familiar properties for probability distributions [7]:

$$
\begin{aligned}
\kappa(\phi) & =\min _{\omega \models \phi} \kappa(\omega) \\
\kappa(\psi \mid \phi) & =\kappa(\psi \wedge \phi)-\kappa(\phi)
\end{aligned}
$$

Typically $\kappa$-values are assumed to be obtained from probabilities, by a form of order of magnitude abstraction in which all probabilities within a given order of magnitude are mapped to the same $\kappa$-value. Following Spohn [13], one can relate a probability $p$ to $\kappa$-value $k$ by:

$$
\epsilon<\frac{p}{\epsilon^{k}} \leq 1
$$

which of course is equivalent to:

$$
\epsilon^{k+1}<p \leq \epsilon^{k}
$$

One procedure to map probabilities into $\kappa$ values is [2]:

1. If $p=0$ then print $\infty$;

2. $k \leftarrow 0$;

3. $p \leftarrow \frac{p}{\epsilon}$;

4. If $p>1$, then print $k$ otherwise $k \leftarrow k+1$;

5. Goto 3;

and an alternative mapping has been suggested by Giang and Shenoy [6]. For this work we assume that either such a mapping has already been applied, or that the $\kappa$-values have been elicited directly — we just assume the existence of a set of $\kappa$-values for the propositions we are interested in.

\section{The $Q R K$ system}

Having introduced the kappa calculus, we can start to introduce the system of argumentation which will use $\kappa$-values. 


\subsection{Basic concepts}

We start with a set of atomic propositions $\mathcal{L}$. We also have a set of connectives $\{\neg, \rightarrow\}$, and the following set of rules for building the well-formed formulas ( $w f f s$ ) of the language.

1. If $l \in \mathcal{L}$ then $l$ is a well-formed simple formula ( $s w f f)$.

2. If $l$ is an swff, then $\neg l$ is an swff.

3. If $l$ and $m$ are $s w f f$ s, then $l \rightarrow m$ is a well-formed implicational formula (iwff)

We denote the set of all swffs which can be derived using $\mathcal{L}$ by $\mathcal{S}_{\mathcal{L}}$, while $\mathcal{I}_{\mathcal{L}}$ denotes the corresponding set of $i w f f s$. The set of $w f f s$ that can be defined using $\mathcal{L}$ is $\mathcal{W}=\mathcal{S}_{\mathcal{L}} \cup \mathcal{I}_{\mathcal{L}}$ may then be used to build up a database $\Delta$ where every item $d \in \Delta$ is a triple $(i: l: s)$ in which $i$ is a token which uniquely identifies the database item (for convenience we will use the letter ' $i$ ' as an anonymous identifier), $l$ is a $w f f$, and $s$ gives information about the degree of belief associated with $l$. In particular we distinguish two cases:

$-l$ is an $s w f f:$ In this case $s$ is the pair expressing the degree of belief associated with $l$ and the degree of disbelief associated with $\neg l$, that is $\langle\kappa(l), \kappa(\neg l)\rangle$;

$-l$ is an iwff: In this case $\rightarrow$ does not represent material implication but that the antecedent of the wff has a probabilistic influence on the consequent. Therefore, the sign $s$ indicates the belief in the consequent given the antecedent. Thus each iwff has associated with it a sign $s$ which is the ordered set of four conditional $\kappa$-values: $\langle\kappa(m \mid l), \kappa(m \mid \neg l), \kappa(\neg m \mid l), \kappa(\neg m \mid \neg l)\rangle$.

Note that there is a notion of direction, similar to that in the directed arcs of probabilistic networks, associated with $i w f f \mathrm{~s}$.

\subsection{The proof theory}

In the previous section we introduced a language for describing belief influences between formulae. For this to be useful we need to give a mechanism for taking sentences in that language and using them to derive new sentences. In particular we need to be able to take formulae with associated $\kappa$-values and use these to derive new formulae and their associated $\kappa$-values. This is done using the consequence relation $\vdash_{Q R K}$ which is defined in Figure 1

The definition is in the form of Gentzen-style proof rules where the antecedents are written above the line and the consequent is written below. The consequence relation operates on a database of the kind of triples introduced in Section 3.1 and derives arguments about formulae from them. The concept of an argument is formally defined as follows:

Definition 1. An argument for a well-formed formula $p$ from a database $\Delta$ is a triple $(p, G, S g)$ such that $\Delta \vdash_{Q R K}(p, G, S g)$ 


$$
\begin{aligned}
& \operatorname{Ax} \frac{}{\Delta \vdash_{Q R K}(S t,\{i\}, S g)}(i: S t: S g) \in \Delta \\
& \neg-\mathrm{E} \frac{\Delta \vdash_{Q R K}(\neg S t, G, S g)}{\Delta \vdash_{Q R K}(S t, G, \operatorname{neg}(S g))} \\
& \neg-\mathrm{I} \frac{\Delta \vdash_{Q R K}(S t, G, S g)}{\Delta \vdash_{Q R K}(\neg S t, G, \operatorname{neg}(S g))} \\
& \rightarrow-\mathrm{E} \frac{\Delta \vdash_{Q R K}(S t, G, S g) \quad \Delta \vdash_{Q R K}\left(S t \rightarrow S t^{\prime}, G^{\prime}, S g^{\prime}\right)}{\Delta \vdash_{Q R K}\left(S t^{\prime}, G \cup G^{\prime}, \operatorname{imp}_{\mathrm{elim}}\left(S g, S g^{\prime}\right)\right)} \\
& \rightarrow-\mathrm{R} \frac{\Delta \vdash_{Q R K}\left(S t^{\prime}, G, S g\right) \quad \Delta \vdash_{Q R K}\left(S t \rightarrow S t^{\prime}, G^{\prime}, S g^{\prime}\right)}{\Delta \vdash_{Q R K}\left(S t, G \cup G^{\prime}, \operatorname{imp}_{\mathrm{rev}}\left(S g, S g^{\prime}\right)\right)}
\end{aligned}
$$

Fig. 1. The consequence relation $\vdash_{Q R K}$

The sign $S g$ of the argument denotes something about the degree of belief associated with the formula $p$, while the grounds $G$ identify the elements of the database used in the derivation of $p$.

To see how the idea of an argument fits in with the proof rules in Figure 1, let us consider the rules $A x$ and $\rightarrow$-E. The first builds an argument from a triple $(i: S t: S g$ ), which has a sign $S g$ and a set of grounds $\{i\}$, where the grounds identify which elements from the database are used in the derivation. This rule is a kind of bootstrap mechanism to allow the elements of the database to be turned into arguments to which other rules can be applied. The second, $\rightarrow$-E, can be thought of as analogous to modus ponens. From an argument for $S t$ and an argument for $S t \rightarrow S t^{\prime}$ it is possible to build an argument for $S t^{\prime}$ once the necessary book-keeping with grounds and signs has been carried out.

\subsection{Combination functions}

In order to apply the proof rules of Figure 1 to build arguments, it is necessary to supply the functions used to combine signs. These are provided in this section.

The rules for handling negation are applicable only to swffs and permit negation to be either introduced or eliminated by altering the sign, for example allowing $(i: a: S g)$ to be rewritten as $\left(i: \neg a: S g^{\prime}\right)$. This leads to the definition of neg:

Definition 2. The function neg: $S g \in\left[0, \infty\left[\times\left[0, \infty\left[\mapsto S g^{\prime} \in[0, \infty[\times[0, \infty[\right.\right.\right.\right.$ is defined as follows:

$$
\begin{aligned}
& \text { If } \quad S g=\left\langle s, s^{\prime}\right\rangle \\
& \text { Then } S g^{\prime}=\left\langle s^{\prime}, s\right\rangle
\end{aligned}
$$

To deal with implication we need two elimination functions impelim and $\mathrm{imp}_{\mathrm{rev}}$, where the former establishes the sign of formulae generated by the rule of inference $\rightarrow-E$, while the latter is used to establish the sign of formulae generated by 
$\rightarrow-\mathrm{R}$. We start by discussing impelim. Let us suppose we have an implicational formula $(i: a \rightarrow b: S g)$ where $S g$ is the quadruple of $\kappa$-values:

$$
\langle\kappa(b \mid a), \kappa(b \mid \neg a), \kappa(\neg b \mid a), \kappa(\neg b \mid \neg a)\rangle
$$

if we have the $s w f f$

$$
(j: a:\langle\kappa(a), \kappa(\neg a)\rangle)
$$

then by applying the rule impelim we can obtain $b$ and the pair $\langle\kappa(b), \kappa(\neg b)\rangle$. In order to do so we have to combine $\langle\kappa(b \mid a), \kappa(b \mid \neg a), \kappa(\neg b \mid a), \kappa(\neg b \mid \neg a)\rangle$ with $\langle\kappa(a), \kappa(\neg a)\rangle$.

Definition 3. The function impelim $: S g \in\left[0, \infty\left[\times\left[0, \infty\left[\times S g^{\prime} \in\left[0, \infty\left[{ }^{4} \mapsto S g^{\prime \prime} \in\right.\right.\right.\right.\right.\right.$ $[0, \infty[\times[0, \infty[$ is defined as follows:

$$
\begin{aligned}
& \text { If } \quad S g=\left\langle s, s^{\prime}\right\rangle \\
& S g^{\prime}=\left\langle r, r^{\prime}, t, t^{\prime}\right\rangle \\
& \text { Then } S g^{\prime \prime}=\left\langle w, w^{\prime}\right\rangle
\end{aligned}
$$

where:

$$
\begin{aligned}
w & =\min \left(r+s, r^{\prime}+s^{\prime}\right) \\
w^{\prime} & =\min \left(t+s, t^{\prime}+s^{\prime}\right)
\end{aligned}
$$

These two equalities are obtained by turning the probabilities in Jeffrey's rule $[8]$ into $\kappa$-values.

The function imp rev is obtained by computing $\operatorname{Pr}(a)$ by manipulating Jeffrey's rule for probabilities with Bayes' rule and then by mapping this expression into kappa calculus.

Definition 4. The function imp rev $: S g \in\left[0, \infty\left[\times\left[0, \infty\left[\times S g^{\prime} \in\left[0, \infty\left[{ }^{4} \mapsto S g^{\prime \prime} \in\right.\right.\right.\right.\right.\right.$ $[0, \infty[\times[0, \infty[$ is defined as follows:

$$
\begin{array}{ll}
\text { If } \quad & S g=\left\langle s, s^{\prime}\right\rangle \\
& S g^{\prime}=\left\langle r, r^{\prime}, t, t^{\prime}\right\rangle \\
\text { Then } & S g^{\prime \prime}=\left\langle w, w^{\prime}\right\rangle
\end{array}
$$

where:

$$
w=\min \left\{s-\min \left(r, r^{\prime}-1\right), r^{\prime}-1-\min \left(r, r^{\prime}-1\right)\right\}
$$

and

$$
w^{\prime}= \begin{cases}\min \left\{s-\min \left(r^{\prime}, r-1\right), r-1-\min \left(r^{\prime}, r-1\right)\right\} & \text { if } w \neq 0 \\ \infty & \text { otherwise }\end{cases}
$$

\subsection{Soundness and completeness}

In order to prove soundness and completeness we first need to capture the kind of relationships that may hold between two formulae: 
Definition 5. A well-formed formula $p$ is said to be a cause of a well-formed formula $q$ if and only if it is possible to identify an ordered set of $i w f f s\{p \rightarrow$ $\left.c_{1}, c_{1} \rightarrow c_{2}, \ldots, c_{n} \rightarrow q\right\}$.

That is, $p$ is a cause of $q$ if it is possible to build up a trail of (causally directed) implications linking $p$ to $q$.

Definition 6. A well-formed formula $p$ is said to be an effect of a well-formed formula $q$ if and only if $q$ is a cause of $p$.

Thus $p$ is an effect of $q$ if it is possible to build up a trail of (causally directed) implications linking $q$ to $p$. Soundness will relate to ensuring that given information about the $\kappa$-value of a particular formula we can compute the correct $\kappa$-value of its causes and effects, and completeness will relate to ensuring that we can compute the $\kappa$-values of all such causes and effects.

Before proceeding to prove soundness and completeness, we need to take into account two problems which can arise when doing evidential reasoning, that is reasoning both in the direction of the implications and in the opposite direction. We are enabled to use evidential reasoning by having included the rule $\rightarrow$ - $R$ in the consequence relation. The first problem arises because when implications are reversed, then the proof procedure can loop and therefore build an infinite number of arguments. This is possible even if we have a single iwff since there is nothing to stop the proof procedure alternately applying $\rightarrow$-E and $\rightarrow$-R forever, building a new argument from each application. However, the problem can be easily solved by introducing the concept of a minimal argument as in [10]:

Definition 7. A minimal argument is an argument in which no iwff appears more than once.

We then reject non-minimal arguments, as we shall see below.

The second problem to deal with is caused by the need to handle conditional independence in the proper way. If proof rules are applied blindly then it is possible to build arguments which do not respect conditional independence. Such arguments would not be valid according to the kappa calculus, so they need to be eliminated. To identify arguments that are invalid because of conditional independence we introduce the notion of $d$-separation from probabilistic networks, suitably modified for $\kappa$-values. However, before proceeding any further we first need to introduce some additional definitions:

Definition 8. A source of an argument $(p, G, S g)$ is an swff from $G$

That is a source of an argument is one of the simple formula which grounds it, and therefore is the head of a chain of implications. In the same way we define the destination of an argument as:

Definition 9. The destination of an argument $(p, G, S g)$ is $p$.

We then define d-separation as follows: 
Definition 10. Two formulae $p$ and $q$ are $d$-separated if for all arguments which have $p$ as their source and $q$ as their destination, there is another formula $r$ such that either:

1. $p$ is a cause of $r, r$ is a cause of $q$, and either $r$ or $\neg r$ is known to be true; or

2. $p$ is an effect of $r, q$ is an effect of $r$, and either $r$ or $\neg r$ is known to be true; or

3. $p$ and $q$ are both causes of $r$ and there is no argument $(r, G, S g)$ such that all the swffs in $G$ are effects of $r$.

We are now in a position to define the subset of all arguments which do not suffer from the two problems we discussed above:

Definition 11. An argument $A=(p, G, S g)$ is invalid if any of the sources of A are d-separated from $p$.

and consequently

Definition 12. An argument $A=(p, G, S g)$ is valid if it is not invalid.

The set of minimal valid arguments are then the problem-free subset of all possible arguments which can be built from some database of triples.

Now, because arguments in $Q R K$ typically only indicate a degree of belief in a formula (rather than indicating that it is true or false), in general there will be several minimal valid arguments concerning it with differing degrees of belief. To combine these, we define a flattening function, and we do this in a way such that only minimal and valid arguments are taken into account. This function, Flat(·) is a mapping from a set of arguments $\mathbf{A}_{S t}^{\Delta}$ for a formula $S t$ built from a particular database $\Delta$ to the pair of that proposition and some overall measure of validity. Thus we have:

$$
\mathbf{A}_{S t}^{\Delta}=\left\{\left(S t, G_{i}, S g_{i}\right) \mid \Delta \vdash_{Q R K}\left(S t, G_{i}, S g_{i}\right)\right\}
$$

and then

$$
\text { Flat : }\left\{A \in \mathbf{A}_{S t}^{\Delta} \mid A \text { is minimal and valid }\right\} \mapsto\langle S t, v\rangle
$$

where $v$ is a single pair of $\kappa$-values, $\langle\kappa(S t), \kappa(\neg S t)$. The value $v$ is then the result of a suitable combination of all the signs of all the arguments for $S t$ :

$$
v=\operatorname{MIN}_{i}\left(\left\{S g_{i} \mid\left(S t, G_{i}, S g_{i}\right) \in \mathbf{A}_{S t}^{\prime \Delta}\right\}\right)
$$

where each $S g_{i}$ is a pair $\langle\kappa(S t), \kappa(\neg S t)\rangle, \mathbf{A}_{S t}^{\prime \Delta}$ is the set of all minimal, valid arguments in $\mathbf{A}_{S t}^{\Delta}$, and the function $\mathrm{MIN}_{i}$ is defined as follows:

$$
\operatorname{MIN}_{i}\left(\left\langle\kappa\left(a_{i}\right), \kappa\left(\neg a_{i}\right)\right\rangle\right)=\left\langle\min _{i} \kappa\left(a_{i}\right), \max _{i} \kappa\left(\neg a_{i}\right)\right\rangle
$$

This definition of the flattening function is motivated by the fact that if we have different arguments, we want to consider the most plausible one - that is we tend to choose the one associated with the most normal world, therefore the one for which holds that $a$ is highly believed while $\neg a$ is highly disbelieved. 
Once the flattening function is established we can use it to provide a procedure to determine the overall procedure for determining the measure of belief in a formula $q$ in which we are interested. This procedure is:

1. Add a triple $(i: p: s)$ for every formula $p$ whose degree of belief is known; 2. Build $\mathbf{A}_{q}^{\Delta}$, the set of all arguments for $q$ using the rules given in Figure 1;

3 . Flatten this set to give $\langle q,\langle\kappa(q), \kappa(\neg q)\rangle\rangle$;

Given the previous definitions it is possible to show that, given information about the degree of belief in (that is the the $\kappa$-value associated with) some formula $p$, the rules of the consequence relation $\vdash_{Q R K}$ may be used to soundly and completely compute arguments concerning the change in the degree of belief associated with the causes and effects of $p$.

Theorem 13. The construction and flattening of arguments in QRK using the rules of $\vdash_{Q R K}$ is sound with respect to the kappa calculus

Proof. The proof is by showing the soundness of the combination functions. For

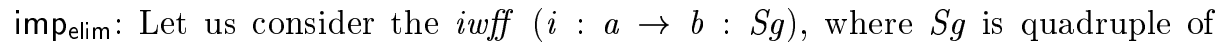
$\kappa$-values:

$$
\langle\kappa(b \mid a), \kappa(b \mid \neg a), \kappa(\neg b \mid a), \kappa(\neg b \mid \neg a)\rangle
$$

From the sign of $a \rightarrow b$ and the sign of $a$, which is $\langle\kappa(a), \kappa(\neg a)\rangle$ we want to be able to calculate the sign of the formula $b$, which is $\langle\kappa(b), \kappa(\neg b)\rangle$. Since $\kappa$-values are equivalent to probabilities, we manipulate Jeffrey's rule [8] and then map into $\kappa$-values in order to obtain $\kappa(b)$. Jeffrey's rule for probabilities is:

$$
\operatorname{Pr}(b)=\operatorname{Pr}(b \mid a) \operatorname{Pr}(a)+\operatorname{Pr}(b \mid \neg a) \operatorname{Pr}(\neg a)
$$

which can be mapped into the kappa calculus expression:

$$
\kappa(b)=\min \{\kappa(b \mid a)+\kappa(a), \kappa(b \mid \neg a)+\kappa(\neg a)\}
$$

In the same way we can use Jeffrey's rule to calculate the probability $\operatorname{Pr}(\neg b)$ from which we obtain the $\kappa$-value formulation for $\kappa(\neg b)$ :

$$
\kappa(\neg b)=\min \{\kappa(\neg b \mid a)+\kappa(a), \kappa(\neg b \mid \neg a)+\kappa(\neg a)\}
$$

since this is exactly the combination function used, impelim is sound. imp rev is slightly less straightforward and requires a few manipulations. This function calculates the sign of a formula $a$ starting from the iwff $(i: a \rightarrow b: s)$ and the $\operatorname{swff}(j: b:\langle\kappa(b), \kappa(\neg b)\rangle)$. We start by proving the formula for $\kappa(a)$. Jeffrey's rule is

$$
\operatorname{Pr}(b)=\operatorname{Pr}(b \mid a) \operatorname{Pr}(a)+\operatorname{Pr}(b \mid \neg a) \operatorname{Pr}(\neg a)
$$

which can be rewritten as:

$$
\operatorname{Pr}(b)=\operatorname{Pr}(b \mid a) \operatorname{Pr}(a)+\operatorname{Pr}(b \mid \neg a)(1-\operatorname{Pr}(a))
$$

which is

$$
\operatorname{Pr}(b)=\operatorname{Pr}(b \mid a) \operatorname{Pr}(a)-\operatorname{Pr}(b \mid \neg a)+\operatorname{Pr}(b \mid \neg a) \operatorname{Pr}(a)
$$


therefore $\operatorname{Pr}(a)$ is obtained as:

$$
\operatorname{Pr}(a)(\operatorname{Pr}(b \mid a)-\operatorname{Pr}(b \mid \neg a))=\operatorname{Pr}(b)-\operatorname{Pr}(b \mid \neg a)
$$

and so

$$
\operatorname{Pr}(a)=\frac{\operatorname{Pr}(b)-\operatorname{Pr}(b \mid \neg a)}{\operatorname{Pr}(b \mid a)-\operatorname{Pr}(b \mid \neg a)}
$$

which mapped into the kappa calculus becomes:

$\kappa(a)=\min \{\kappa(b)-\min [\kappa(b \mid a), \kappa(b \mid \neg a)-1], \kappa(b \mid \neg a)-1-\min [\kappa(b \mid a), \kappa(b \mid \neg a)-1]\}$

where the absolute value is added to make sure $\kappa(a) \geq 0$. In the same way the expression for $\kappa(\neg a)$ can be computed, thus obtaining:

$\kappa(\neg a)=\min \{\kappa(b)-\min [\kappa(b \mid \neg a), \kappa(b \mid a)-1], \kappa(b \mid a)-1-\min [\kappa(b \mid \neg a), \kappa(b \mid a)-1]\}$

neg is quite straightforward, following directly from the definition. The soundness of the flattening function can be proved by demonstrating that if we have two different arguments for a formula $c$, one from $a$ to $b$ and then to $c$ and the second from $d$ to $b$ to $c$ then the degree of belief which results from flattening the two arguments is the same that would be computed were there only one argument from $a$ and $d$ in combination to $b$, and then from $b$ to $c$, where the combination is disjunctive, making something like the usual Noisy-Or assumption. In the first case we have the following chain of formulae:

1. $a \rightarrow b \rightarrow c$

2. $d \rightarrow b \rightarrow c$

and the two swffs $(i: a:\langle\kappa(a), \kappa(\neg a)\rangle)$ and $(j: d:\langle\kappa(d), \kappa(\neg d)\rangle)$ Let us denote with $\kappa_{1}(c)$ the degree of belief associated with the first argument and with $\kappa_{2}(c)$ the degree of belief associated with the second one. By applying $\rightarrow-E$ twice we can compute:

$$
\begin{aligned}
\kappa_{1}(c)=\min \{ & \kappa(c \mid b)+\min [\kappa(b \mid a)+\kappa(a), \kappa(b \mid \neg a)+\kappa(\neg a)], \\
& \kappa(c \mid \neg b)+\min [\kappa(\neg b \mid a)+\kappa(a), \kappa(\neg b \mid \neg a)+\kappa(\neg a)]\}
\end{aligned}
$$

where we have used the following substitutions:

$$
\begin{gathered}
\kappa(b)=\min \{\kappa(b \mid a)+\kappa(a), \kappa(b \mid \neg a)+\kappa(\neg a)\} \\
\kappa(\neg b)=\min \{\kappa(\neg b \mid a)+\kappa(a), \kappa(\neg b \mid \neg a)+\kappa(\neg a)\}
\end{gathered}
$$

Analogously we can compute $\kappa_{2}(c)$ as:

$$
\begin{aligned}
\kappa_{2}(c)= & \min \{\kappa(c \mid b)+\min [\kappa(b \mid d)+\kappa(d), \kappa(b \mid \neg d)+\kappa(\neg d)], \\
& \kappa(c \mid \neg b)+\min [\kappa(\neg b \mid d)+\kappa(d), \kappa(\neg b \mid \neg d)+\kappa(\neg d)]\} .
\end{aligned}
$$

In order to compute the degree of belief associated with $c$ we need to flatten the two arguments by using the flattening function defined above. If we flatten them the resulting degree of belief will be

$$
\left\langle\min \left(\kappa_{1}(c), \kappa_{2}(c)\right), \max \left(\kappa_{1}(\neg c), \kappa_{2}(\neg c)\right)\right\rangle
$$


that is for $c$ the overall $\kappa$-value is:

$$
\min \{\min [\kappa(c \mid b)+\alpha, \kappa(c \mid \neg b)+\beta], \min [\kappa(c \mid b)+\gamma, \kappa(c \mid \neg b)+\delta]\}
$$

where:

$$
\begin{aligned}
\alpha & =\min [\kappa(b \mid a)+\kappa(a), \kappa(b \mid \neg a)+\kappa(\neg a)] \\
\beta & =\min [\kappa(\neg b \mid a)+\kappa(a), \kappa(\neg b \mid \neg a)+\kappa(\neg a)] \\
\gamma & =\min [\kappa(b \mid d)+\kappa(d), \kappa(b \mid \neg d)+\kappa(\neg d)] \\
\delta & =\min [\kappa(\neg b \mid d)+\kappa(d), \kappa(\neg b \mid \neg d)+\kappa(\neg d)]
\end{aligned}
$$

This can be rewritten as:

$$
\min \{\kappa(c \mid b)+\min (\alpha, \gamma), \kappa(c \mid \neg b)+\min (\beta, \delta)\}
$$

This result is the same as the degree of belief which would be computed were the degree of belief in $b$ first computed from a disjunctive dependence on $a$ and $d$ and the result then propagated to $c$. Something very similar can be carried out for the $\kappa$-value of $\neg c$, but with max in place of the outer min, thus proving that $Q R K$ flattens arguments soundly. This concludes the proof.

Having proved the soundness we can move on to prove completeness, but before giving such a proof we need to define what we mean by completeness.

Definition 14. The construction and flattening of arguments is said to be complete with respect to some formula $p$ if it is possible to use that system to compute all the $\kappa$-values of all the effects of $p$, all the causes of $p$ and all the causes and effects of all the causes and effects of $p$.

With this definition it is now possible to state and prove the following theorem:

Theorem 15. The construction and flattening of arguments in QRK is complete with respect to any formula.

Proof. The proof follows from the definition of $\vdash Q R K$, that is the $\kappa$-value of all the causes and effects of any well-formed formula $p$ which may be stated in $Q R K$ can be made by the application of the appropriate proof rules. In proving this we need to distinguish proof of completeness for causes from those for effects. We start from the latter. Let us consider the addition of the triple $(i: p$ : $(\kappa(p), \kappa(\neg p)))$ where $p$ contains no negation symbols, to a database that contains only formulae without negation symbols. We can have two types of of effect of $p$ : The first are consequents of implications in which $p$ forms the antecedent while the second are those effects that are related to $p$ by two or more implications. In the first case the $\kappa$-values associated with the formula can be computed by applying the proof rule $\rightarrow$-E. In the latter case the degree of belief associated with the formula may be obtained by recursively applying the $\rightarrow$-E rule. 
Analogously, we can recognise two types of causes of $p$, those which are antecedents of implications where $p$ is the consequent and those which are causes that are related to $p$ by two or more implications. In the first case the $\kappa$-value associated with the formula is computed by $\rightarrow$ - $R$ while in the second case the $\kappa$-value may be obtained by recursively applying $\rightarrow$-R.

Applying both $\rightarrow-\mathrm{E}$ and $\rightarrow-\mathrm{R}$ recursively is sufficient to ensure completeness for situations without negation, and the appropriate use of the rules $\neg$-I and $\neg$-E make it possible to deal with situations in which the negation symbol appears.

\section{Example}

Let us suppose we have the following information about the health of a friend. The event that our friend has a cold $(\mathrm{C})$ increases the belief that she is sneezing (S). But also the event $\mathrm{R}$ that she has an allergic reaction increases the belief that she is sneezing. The event $\mathrm{T}$, that our friend has taken some antihistamine, however, reduces the belief that she is sneezing, while the event that she has an allergic reaction $\mathrm{R}$ increases the belief that she has taken an antihistamine. The event $\mathrm{A}$, that our friend is allergic to cats increases the belief that she might have an allergic reaction. This information may be represented as:

$$
\begin{aligned}
& (r 1: C \rightarrow S:\langle\kappa(S \mid C)=0, \kappa(\neg S \mid C)=1, \kappa(S \mid \neg C)=2, \kappa(\neg S \mid \neg C)=1\rangle) \quad \Delta \\
& (r 2: R \rightarrow S:\langle\kappa(S \mid R)=0, \kappa(\neg S \mid R)=1, \kappa(S \mid \neg R)=2, \kappa(\neg S \mid \neg R)=1\rangle) \\
& (r 3: T \rightarrow S:\langle\kappa(S \mid T)=2, \kappa(\neg S \mid T)=1, \kappa(S \mid \neg T)=1, \kappa(\neg S \mid \neg T)=1\rangle) \\
& (r 4: R \rightarrow T:\langle\kappa(T \mid R)=1, \kappa(\neg T \mid R)=1, \kappa(T \mid \neg R)=4, \kappa(\neg T \mid \neg R)=1\rangle)
\end{aligned}
$$

If we believe that our friend is having an allergic reaction, then we can add the following fact to $\Delta$ :

$$
(f 1: R:\langle\kappa(R)=1, \kappa(\neg R)=3\rangle) .
$$

Adding this fact permits us to build two minimal, valid arguments concerning our friend taking antihistamine:

$$
\left.\Delta \vdash_{Q R K}(T,\{f 1, r 4\},\langle\kappa 1(T)=3, \kappa 1(\neg T)=3)\rangle\right),
$$

by applying $\rightarrow$-E once while if we first apply $\rightarrow-E$ and then $\rightarrow-\mathrm{R}$ we obtain:

$$
\Delta \vdash_{Q R K}(T,\{f 1, r 2, r 3\},\langle\kappa 2(T)=0, \kappa 2(\neg T)=\infty\rangle)
$$

By flattening these combine to give the pair $\langle T,\langle\kappa(T)=0, \kappa(\neg T)=\infty\rangle\rangle$ to indicate that the event that our friend is not taking antihistamine warrants a much greater degree of disbelief than the event that she is taking antihistamine.

\section{Conclusions}

In this paper we have presented $Q R K$, a system of argumentation in which uncertainty is handled using infinitesimal probability values, in particular values 
from the kappa calculus. The use of $\kappa$-values means that the system can be used when probabilistic knowledge of a domain is incomplete, and this makes it applicable to a wider range of situations with respect to systems based on complete probabilistic information. The system associates a $\kappa$-value with every logical formula, and combines these values as arguments are built in a way which is sound with respect to the kappa calculus. Thus the arguments which can be constructed in $Q R K$ come complete with an order of magnitude estimate of the probability of the formula supported by the argument, and the system thus supports qualitative probabilistic reasoning.

\section{References}

1. S. Benferhat, D. Dubois, and H. Prade. Argumentative inference in uncertain and inconsistent knowledge bases. In Proceedings of the 9th Conference on Uncertainty in Artificial Intelligence, 1993.

2. A. Darwiche and M. Goldszmidt. On the relation between kappa calculus and probabilistic reasoning. In Proceedings of the 10th Conference on Uncertainty in Artificial Intelligence, 1994.

3. M. J. Druzdzel. Probabilistic reasoning in decision support systems: from computation to common sense. PhD thesis, Carnegie Mellon University, 1993.

4. P. M. Dung. On the acceptability of arguments and its fundamental role in nonmonotonic reasoning and logic programming. In Proceedings of the 13th International Conference on Artificial Intelligence, 1993.

5. J. Fox. A unified framework for hypothetical and practical reasoning (2): lessons from clinical medicine. In Proceedings of the Conference on Formal and Applied Practical Reasoning, 1996.

6. P. H. Giang and P. P. Shenoy. On transformations between probability and Spohnian disbelief functions. In K. B. Laskey and H. Prade, editors, Proceedings of the 15th Conference on Uncertainty in Artificial Intelligence, pages 236-244, San Francisco, CA, 1999. Morgan Kaufmann.

7. M. Goldszmidt and J. Pearl. Qualitative probabilistic for default reasoning, belief revision, and causal modelling. Artificial Intelligence, 84(1-2):57-112, 1996.

8. R. Jeffrey. The logic of decision. University of Chicago Press, Chicago, IL, 2nd edition, 1983.

9. R. Loui. Defeat among arguments: a system of defeasible inference. Computational Intelligence, 3:100-106, 1987.

10. S. Parsons. A proof theoretic approach to qualitative probabilistic reasoning. International Journal od Approximate Reasoning, 19:265-297, 1998.

11. S. Parsons. Qualitative approaches to reasoning under uncertainty. MIT Press, (to appear), Cambridge, MA, 1998.

12. J. Pearl. From conditional oughts to qualitative decision theory. In Proceedings of the 9th Conference on Uncertainty in Artificial Intelligence, 1993.

13. W. Spohn. Ordinal conditional functions: A dynamic theory of epistemic states. In W.L. Harper and B. Skyrms, editors, Causation in Decision, Belief Change, and Statistics, volume 2, pages 105-134. 1987.

14. M. P. Wellman. Formulation of tradeoffs in planning under uncertainty. Pitman, London, 1990.

15. N. Wilson. An order of magnitude calculus. In Proceedings of the 11th Conference on Uncertainty in Artificial Intelligence, 1995. 